\title{
Elección de acceso vascular en un paciente tetrapléjico en hemodiálisis: un obstáculo a superar
}

\author{
Engracia Martínez Rodríguez, Covadonga Díaz García, Ana María Mon Rodríguez, Alejandra Méndez González
}

Unidad de Hemodiálisis del Área de Gestión del Servicio de Nefrología del Hospital Central Universitario de Asturias. España

\section{Resumen}

Los accesos vasculares son uno de los pilares fundamentales de la hemodiálisis. El acceso ideal debe proporcionar un flujo adecuado de sangre, ser de larga duración, permitir un abordaje fácil y sin complicaciones.

La elección del acceso debe ser individualizada para cada paciente, según diversos aspectos como historia clínica, mapa vascular o preferencias del paciente. La fístula arteriovenosa autóloga es el acceso que más se asemeja a las cualidades del acceso ideal, siendo acceso de primera elección.

Se restringen los catéteres venosos centrales tunelizados para situaciones con imposibilidad de conseguir accesos de primera elección.

El objetivo de este artículo es mostrar la experiencia de un paciente tetrapléjico en HD, en la búsqueda y elección del acceso vascular.

La elección del acceso más adecuado en casos como este tiene importantes connotaciones añadidas, por lo que la decisión no siempre es fácil. Por un lado, los accesos de primera elección pueden limitar o condicionar el quehacer diario de pacientes tetrapléjicos/parapléjicos, y, por otro lado, los catéteres elevan sustancialmente el riesgo de infecciones y la morbimortalidad.

PALABRAS CLAVE: acceso vascular; fístula arterio-venosa autóloga, catéter venoso tunelizado, tetraplejia/paraplejia.

Correspondencia:

Engracia Martínez Rodríguez

C/. Puerta Europa, 2. $4^{\circ} \mathrm{C} .33011$ Oviedo

E-mail: martinezrodriguezengracia530@gmail.com

\section{Choice of vascular access in a tetraplegic patient on hemodialysis: an obstacle to overcome}

\section{Summary}

Vascular accesses are one of the mainstays of hemodialysis. The ideal access should provide an adequate blood flow, be long-lasting, allow easy and uncomplicated handling.

The choice of access should be individualized for each patient, according to various aspects such as clinical history, vascular map or patient preferences. Autologous arteriovenous fistula is the access that most resembles the qualities of ideal access, being the first-line access.

Tunneled central venous catheters are restricted for situations with inability to achieve first-line access.

The aim of this article is to show the experience of a tetraplegic patient in HD, in the search and selection of his vascular access.

The choice of the most appropriate access in such cases has important added connotations, so the decision is not always easy. On the one hand, the first-line access may limit or condition the daily work of tetraplegic / paraplegic patients, and, on the other hand, catheters substantially increase the risk of infections and morbimortality.

KEYWORDS: vascular access; autologous arteriovenous fistula; tunneled venous catheter; tetraplegia/ paraplegia.

\section{Introducción}

Los Accesos Vasculares (AV) son uno de los pilares fundamentales de la Hemodiálisis (HD). Las cualidades del AV ideal son: proporcionar un flujo adecuado de sangre 
asegurando una diálisis eficaz, ser de larga duración, sin ocasionar complicaciones y permitir un abordaje fácil y seguro ${ }^{1}$.

Las Fístulas Arterio-Venosas Autólogas o Nativas (FAVn) son los AV que más se acercan a cumplir los requisitos del acceso ideal y por ello, son los accesos de primera elección. La selección del AV ha de ser individualizada y se han de tener en cuenta diferentes variables como las preferencias del paciente, los factores relacionados con la propia comorbilidad del paciente, o el estudio del mapa vascular del paciente ${ }^{1,2}$.

La localización debe ser lo más distal posible para no limitar la realización de accesos posteriores, y se debe tener siempre presente que pueden aparecer complicaciones como edemas en el brazo portador de la FAV por estenosis venosas profundas, y que la FAV puede comprometer la vascularización distal, ocasionando un síndrome isquémico de hipoperfusión distal o síndrome de robo de fístula, que aun siendo poco frecuente (1-1,5\%), puede derivar en serias limitaciones para la funcionalidad del miembro afectado así como para el confort del paciente y para la viabilidad de la propia FAV ${ }^{1-4}$.

Además, ser portador de una FAV implica que el paciente ha de seguir una serie de recomendaciones para su cuidado, pudiendo esto significar un hándicap para algunos pacientes ${ }^{5,6}$.

Cuando no se puede conseguir una FAVn adecuada, se realiza la conexión arterio-venosa mediante el implante de un injerto tubular de material sintético, el politetrafluoretileno (FAVp). Los injertos tienen como ventajas una superficie mayor para la punción, una canalización más fácil y un corto tiempo de maduración. El mayor inconveniente es una permeabilidad menor, que las FAVn a largo plazo siendo una opción más cara y con mayor morbilidad para el paciente ${ }^{1-4}$.

Una vez agotadas las posibilidades de primera elección se recurre a los Catéteres Venosos Centrales Tunelizados (CVCT). Son candidatos de CVCT pacientes con: arteriopatía periférica severa, hipotensión crónica severa, una esperanza de vida inferior a 6 meses, miocardiopatía grave, en espera de trasplante renal de vivo, insuficiencia de red vascular e imposibilidad total de realizar cualquier tipo de FAV o que éstas estén en fase de maduración o con fracaso renal agudo o crónico agudizado en el que se precise realizar Hemodiálisis con urgencia. Con los CVCT se incrementan sustancialmente la morbi-mortalidad (10-20\%) respecto a accesos de primera elección (la tasa de mortalidad asciende un 53\% frente a FAVn y un $18 \%$ frente FAVp), el riesgo de hospitalización, que aumenta un $68 \%$ respecto a FAVn (estancia media de 7 días), el coste médico y la tasa de infecciones. Los accesos vasculares son el origen del 50\%-80\% de las bacteriemias en diálisis. De ellos, los CVCT son el origen de la mayor parte y suelen precisar su retirada y sustitución ${ }^{13,4,7-10}$.

El objetivo de este artículo es mostrar la experiencia de un paciente tetrapléjico en $\mathrm{HD}$, en la búsqueda y elección del AV más adecuado a sus circunstancias.

Para la elaboración de este trabajo se ha revisado retrospectivamente la historia clínica de un paciente de la unidad de HD del Hospital Universitario Central de Asturias (HUCA), y se ha obtenido la aprobación del comité de ética de dicho hospital, así como el consentimiento informado del paciente.

\section{Caso Clínico}

Paciente de 52 años, tetrapléjico por lesión medular a nivel C6-C7 desde los 22 años, tras un accidente de tráfico en 1987, y que conservó la movilidad y funcionalidad de Miembros Superiores (MMSS).

En 2009 se le extirpa el riñón derecho por un hipernefroma, y en agosto del 2014 se le realiza una nefrectomía izquierda por cáncer células claras grado I de Fuhrman, precisando iniciar tratamiento sustitución renal de forma inmediata, e incluyéndose en el programa de hemodiálisis. En agosto del 2017 fallece a causa de hematoma subdural.

Se trataba de una persona llena de energía que asumía su situación positivamente. Independiente para la mayoría de actividades de la vida diaria (comer, afeitarse...) precisando ayuda parcial en relación con la movilidad y los desplazamientos. Fue provisto de diversos dispositivos y de adaptaciones arquitectónicas en su domicilio, además de dos sillas eléctricas con distinta potencia y estructura que le permitían una gran autonomía, y una silla manual.

Al inicio en HD, se le colocó un CVCT en Yugular Interna Superior Derecha, con el que se consiguió una eficacia de diálisis relativamente baja, con unos valores medios de $324 \mathrm{ml} / \mathrm{min}$ de flujo sanguíneo, 42 I de KT y 1,28 de KT/V y recirculación $8,4 \%$. Realizaba sesiones de $4 \mathrm{~h}$ de duración, tres veces a la semana con un diali- 
zador capilar de membrana helixona de alta permeabilidad con superficie de $1,8 \mathrm{~m}^{2}\left(\mathrm{FX} 80^{\circledR}\right)$.

En marzo del 2015 (a los seis meses de comienzo en $H D$ ) se crea una $F A V n$ radiocefálica en miembro superior izquierdo (no dominante) la cual a los 2 meses de su realización, durante la fase de maduración, se trombosó, sin llegar a usarse.

En julio de 2015, presentó un episodio de shock séptico con fracaso multiorgánico, relacionado con una bacteriemia provocada por el catéter, que supuso un grave compromiso para su vida. Permaneció 14 días hospitalizado, 7 de ellos en la UCI, con intubación orotraqueal y Ventilación Mecánica. Manifestaba un estado de intensa agitación, alteración de la conducta y bajo nivel de conciencia. Para su tratamiento de HD se insertó un Catéter Venoso Central temporal (CVCt) en subclavia izquierda.

Se instauró precozmente tratamiento empírico hasta resultados de cultivos, con piperacilina-tazobactan, linezolid y amikacina. Los hemocultivos y el cultivo del orificio del catéter fueron positivos a Staphilococcus Aureus sensible a meticilina. Su estado general fue mejorando, con recuperación del estado cognitivo y nivel de conciencia, permitiendo desintubación y paso a planta. Con el antibiograma se ajusta tratamiento con amoxicilina/clavulánico. La fiebre siguió persistiendo, optando por la retirada del CVCT e insertando un CVCt femoral derecho para la realización de HD. Este acceso se trombosó y se canalizó nuevo en femoral izquierdo hasta implantación del tunelizado.

El nuevo CVCT fue colocado en agosto del 2015 en yugular interna derecha, a la espera de la realización de su segunda FAV. En ese momento realizaba Hemodiafiltración Postdilución, de 4 1/2 horas de duración con el mismo dializador $\left(\mathrm{FX} 80^{\circledR}\right.$ ).

En diciembre de 2015 se le realiza una nueva FAVn húmero-cefálica en Miembro Superior Izquierdo (MSI), que maduró adecuadamente y se estuvo utilizando 3 meses, presentando problemas desde el comienzo, como punciones dificultosas o roturas del acceso por espasmos, precisando en numerosas ocasiones la conexión al catéter de una o las dos ramas. Finalmente, terminó trombosándose. Con estas incidencias, las cifras de eficacia dialítica fueron aún menores que con el CVCT.

El 30 de noviembre del 2016 se le interviene para realizar la tercera FAV, húmero-cefálica en miembro superior derecho (dominante). Al quinto día de su realización acude a urgencias por edema importante en dicho miembro. Se realiza una fistulografía en la que se aprecia buena permeabilidad de vena cefálica y estenosis severa del tronco braquiocefálico venoso derecho en relación con el CVCT yugular. Se le realiza una angioplastia en vena cava superior. A los 15 días acude nuevamente con fiebre, alteración de la conducta, espasmos generalizados y aumento del edema del miembro de la fístula, hematoma, enrojecimiento y calor. Se supone sobreinfección de FAV, que sigue con thrill. Se instaura tratamiento empírico hasta resultados de hemocultivos.

Una vez tratada y curada la infección en el acceso, el edema siguió persistiendo, con los consiguientes obstáculos para su vida diaria. Finalmente, y sin haberse utilizado, en abril de 2017 se decide cerrar el acceso, tras lo que el miembro recupera su morfología y funcionalidad habitual. Ante estas circunstancias, se baraja la posibilidad de colocar una FAVp.

En julio del 2017 es incluido en la lista de trasplante renal y al día siguiente es Ilamado para recibir un órgano de donante cadáver. Ya en quirófano y anestesiado, al realizar el sondaje vesical pertinente, se observa orina piúrica, anulándose el trasplante. En el urinocultivo creció una Pseudomona, que fue tratada con amikacina.

En la sesión del 22 de agosto del 2017 acude a la unidad ansioso y agitado, con movimientos estereotipados, disnea y opresión centrotorácica. Su nivel de conciencia fue disminuyendo por lo que realiza una Tomografía Axial Computerizada urgente en el que se aprecia hematoma subdural con sangrado activo en ese momento. Dado el mal pronóstico se desestima actitud quirúrgica siendo éxitus ese mismo día.

\section{Discusión}

Tanto en las sesiones de HD como fuera de ellas, eran frecuentes las hipotensiones severas, factor de riesgo para la viabilidad y funcionalidad de los accesos, que pueden llegar a trombosarse 0 a no permitir flujos de sangre adecuados. Además de ser una complicación aguda propia de la HD, la hipotensión arterial en las personas con tetraplejias o paraplejias altas se debe a la respuesta simpática alterada que se traduce en falta de vasoconstricción compensatoria a nivel del lecho esplácnico y del lecho vascular en el sistema muscu- 
loesquéletico. Lo anterior, asociado a estasis venosa en miembros inferiores y a la disminución de la actividad muscular que reduce el retorno venoso, explica las bajas tensiones ${ }^{11}$.

También aparece con mayor frecuencia en estos pacientes la hipotensión ortostática que se define como una bajada de la tensión sistólica mayor de $20 \mathrm{mmHg}$ y/o una disminución de la tensión diastólica mayor de 10 $\mathrm{mmHg}$ al pasar de una posición supina a la vertical (sedestación o bipedestación) ${ }^{11}$.

Asimismo, la espasticidad propia de las lesiones medulares, y definida como un trastorno motor caracterizado por el incremento de los reflejos tónicos con aumento del tono muscular que afectan a tronco y miembros, puede suponer complicaciones durante las sesiones, ya que los movimientos involuntarios pueden ocasionar movilizaciones de agujas con evidente riesgo de extravasación y generación de hematomas lesionando el endotelio vascular además de poder provocar desconexiones accidentales de agujas ${ }^{11}$.

Durante los periodos en los que fue portador de FAV, su vida se encontró limitada en mayor medida por temor a perjudicar al acceso. El gran apoyo familiar, tanto físico como psicológico que el paciente recibía, ha sido sumamente importante para afrontar todos obstáculos.

Hasta el momento de su fallecimiento se estuvo dializando con el Catéter Venoso Central Tunelizado insertado tras el episodio infeccioso del primer CVCT. La eficacia de la HD en los últimos 6 meses (marzo-agosto 2017) conseguida con este acceso se refleja en los siguientes datos medios: flujo sanguíneo de 346,8 $\mathrm{ml} / \mathrm{min} ; \mathrm{KT}$ de 46,5 I; KT/V de 1,4; recirculación de 9,5\%; volumen de sustitución con Online de 20,02 I. Ocasionalmente, se dio algún episodio disfuncional precisando la inversión de ramas y/o el tratamiento fibrinolítico con uroquinasa, con repercusión en la eficacia de la diálisis durante esas sesiones y en la media de los datos anteriores.

Aunque las Guías de Práctica Clínica señalan a las FAVn como los accesos vasculares de primera elección para realizar HD, y se dejan los CVCT para circunstancias especiales ${ }^{1-4}$. Hemos descrito la situación de un paciente con Insuficiencia Renal Crónica Terminal, tetrapléjico, portador, desde su inicio en el programa de HD de un CVCT, que ha sido el origen de un grave episodio de infección sistémica con elevado riesgo vital, y que además, también fue portador de tres FAVn, dos en miembro no dominante, y otra en miembro dominante, con muy corta vida funcional y escasa eficiencia.
Suponemos que el desempeño de las actividades cotidianas en personas paraplégicas/tetrapléjicas puede repercutir en la supervivencia de la FAV, especialmente si está localizada en el miembro dominante (esfuerzos, movimientos bruscos). Al mismo tiempo, hemos visto que la funcionalidad de los miembros superiores (MMSS) en personas paraplégicas/tetrapléjicas puede verse comprometida por algunas de las complicaciones de las FAV (robo vascular, isquemia, edemas...), y las precauciones y cuidados que exigen estos accesos interfieren en el desarrollo de las actividades habituales.

Por tanto, podemos afirmar que la elección del acceso vascular no siempre es una fácil decisión y este caso es un claro ejemplo de ello, ya que por un lado, los accesos de primera elección han limitado o condicionado el quehacer diario de un paciente muy limitado previamente, y por otro lado, el CVCT puso en riesgo su vida, a causa de una grave infección.

\section{Agradecimientos}

Nuestro agradecimiento a José Luis Núñez Quinteiro y a su familia por la aprobación y colaboración en la realización de este trabajo. Sea este un pequeño homenaje hacia un hombre que, a pesar de todos los obstáculos a los que fue enfrentándose, siempre se mostró positivo y con ganas de luchar.

\section{El autor declara que no hay conflicto de interés.}

Recibido: 30 noviembre 2017

Revisado: 15 enero 2018

Modificado: 28 enero 2018

Aceptado: 30 enero 2018

\section{Bibliografía}

1. Guías de Acceso Vascular en Hemodiálisis. Sociedad Española Enfermería Nefrológica. [Internet] 2004. [Consultado 30 octubre 2017). Disponible en: http:// www.seden.org/files/rev49_1.pdf.

2. Ibeas J, Roca R, Vallespin J, Moreno T, Moñux G, Martí-Montós A et al. Guía Clínica Española del Acceso Vascular para Hemodiálisis. [Internet] 2017. (Acceso 30 de octubre de 2016).Disponible en:http:// 
revistanefrologia.com/es-publicacion-nefrologiaarticulo-guia-clinica-espanola-del-acceso-vascularhemodialisis-S0211699517302175.

3. A. Galera-Fernández a, M.T. Martínez-de Merlo, B. A. Ochando-García. Accesos vasculares para hemodiálisis: cuidados de enfermería. Angiología 2005; 57. S 165-167.

4. National Kidney Foundation. NKF K/DOQI: Clinical practice guidelines for vascular access: update 2000. Am J Kidney Dis. 2001 Jan;37(1 Suppl 1):137-81.

5. Arrieta J, Ruiz Camos I, Martínez de Merlo M, Gallego Beuter JJ, Herrero Calvo JA, Rodríguez Hernández $\mathrm{J}$ et al. Guía de acceso vascular en hemodiálisis. Angiología 2005;57(2):119-207.

6. National Kydney Foundation. KDOQI Clinical Practice Guidelines and Clinical Practice Recommendations for 2006 Updates: Hemodialysis Adequacy, Peritoneal Dialysis Adequacy and Vascular Access. Am J Kidney Dis 2006; (Suppl 1) (48):S1-S322.

7. Gruss E, Portolés J, Tato A, López-Sánchez P, Velayos $\mathrm{P}$, Gago MC et al. Repercusiones clínicas y económicas del uso de catéteres tunelizados de hemodiálisis en un área sanitaria. Nefrología 2009;29 (2):123-129.

8. Aroca A, Lopez de Rodas M, López-Montes A, Martínez-Lorenzo MC, Martínez-Mora MJ, Martínez-Villaescusa $M$ et al. Protocolo de catéter venoso central para hemodiálisis, manejo y prevención de bacteriemias. Servicio. Unidad de hemodiálisis. Hospital General Universitario de Albacete, [Internet] 2013, revisado 2015. [Consultado mayo 2017]. Disponible en: https://www.chospab.es/publicaciones/protocolosEnfermeria/documentos/4eabc6dd46b963e97ele9165654563f2.pdf.
9. Reula A, Martínez-Olivares E, Martínez-Mas A, Villaro J, Sainz M, Ortells R et al. Dificultad para conseguir un acceso vascular para hemodiálisis. Caso clínico: 9 años en hemodiálisis, 15 accesos vasculares. Enferm Nefrol 2012: Julio-Septiembre; 15 (3): 219/221.

10. Aguinaga A, Del Pozo JL. Infección asociada a catéter en hemodiálisis: diagnóstico, tratamiento y prevención. NefroPlus 2011;4:1-10.

11. Guía para el manejo integral del paciente con LM crónica. Aspaym. Madrid [Internet] 2015.pp:39-55 [Consultado 30 octubre 2017]. Disponible en: http:// www.aspaymmadrid.org/images/documentos/pdf/ guia-manejo-integral-2013.pdf.

Este artículo se distribuye bajo una Licencia Creative Commons Atribución-NoComercial 4.0 Internacional. https://creativecommons.org/licenses/by-nc/4.0/

\section{Open Access (c) $(9)$ ()}

